\title{
Molecular Origin of Superlubricity between Graphene and Highly Hydrophobic Surface in Water
}

\author{
Jinjin Li ${ }^{\# *}$,Wei Cao ${ }^{\#}$, Jianfeng Li, Ming Ma*, and Jianbin Luo
}

State Key Laboratory of Tribology, Tsinghua University, Beijing, 100084, China

1. XPS result of the silica probe after the self-assemble of FDTS molecules

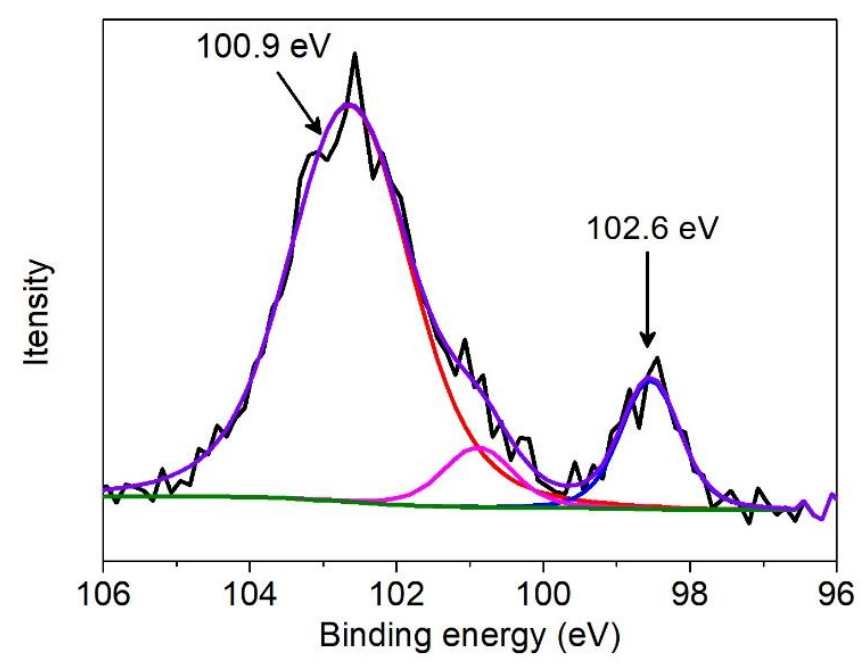

Figure S1 XPS spectrum of Si 2p on the top region of SAFM probe after the self-assemble of FDTS.

The two peaks are from the bonds $\mathrm{Si}-\mathrm{O}(102.6 \mathrm{eV})$ and $\mathrm{Si}-\mathrm{C}(100.9 \mathrm{eV})$, respectively.

2. Density and structure factor of the double-layered water structure confined between SAFMs and graphene 

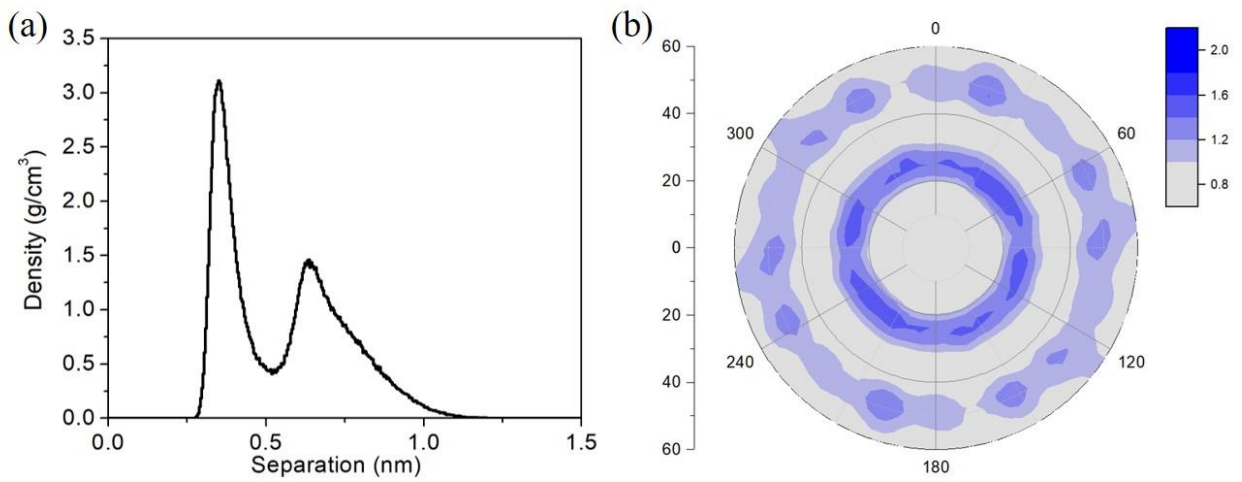

Figure S2 (a) Density as a function of separation for the double-layered water structure confined between SAFMs and graphene. (b) Two-dimensional structure factor of the double-layered water structure confined between SAFMs and graphene.

3. Shear stress as a function of normal load

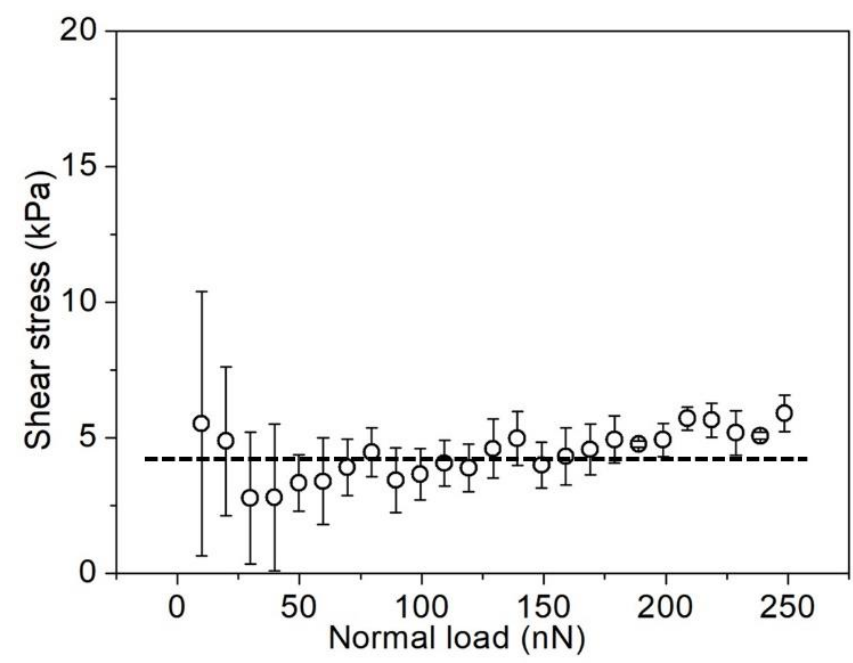

Figure S3 Shear stress as a function of normal load when the SAFM probe slides on the graphene surface across pure water with a constant sliding velocity of $4 \mu \mathrm{m} / \mathrm{s}$. The shear stress was obtained by $\tau=F_{s} / A$, where $F_{s}$ is the measured frictional force and $A$ is the contact area under the given load, which could be estimated by the Hertz contact mechanics. 
4. Shear stress as a function of sliding velocity

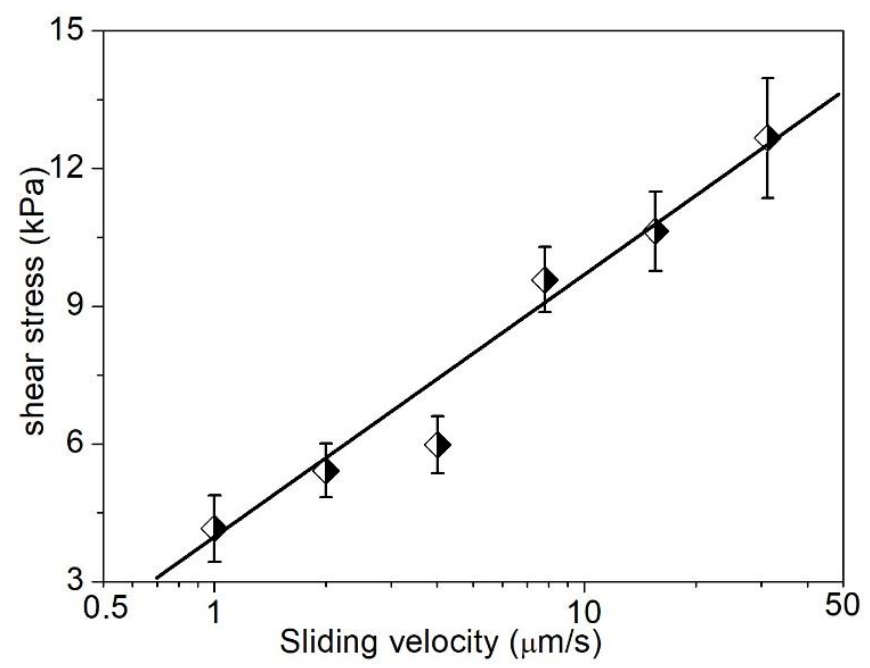

Figure S4 Shear stress as a function of sliding velocity when the SAFM probe slides on the graphene surface across pure water under a constant normal load of $99 \mathrm{nN}$. The shear stress was obtained by $\tau$ $=F_{S} / A$, where $F_{S}$ is the measured frictional force under different sliding velocities, and $A$ is the contact area under the normal load of $99 \mathrm{nN}$. 